\title{
Modelagem computacional de ventosas automáticas de duplo efeito com abertura e fechamento não instantâneos
}

\author{
Computational modeling of double effect automatic air valves opening \\ and closing is not instantaneous
}

\author{
Marcus Vinícius Sousa Rodrigues | Marco Aurélio Holanda de Castro | Marisete Dantas de Aquino
}

Data de entrada: 25/07/2012 | Data de aprovação: 05/07/2013

DOI: http://dx.doi.org/10.4322/dae.2014.005

\section{Resumo}

Durante fenômenos transitórios podem ocorrer pressões muito baixas nos trechos mais elevados de uma tubulação, ocorrendo o acúmulo de ar que é arrastado pela água ocasionando sérios danos ao sistema. Os dispositivos mais utilizados para o alívio do transiente hidráulico (golpe de aríete) trabalham com ar, dentre eles, os mais utilizados são as ventosas automáticas, foco deste estudo. Neste trabalho foram feitas simulações do transiente hidráulico para verificar a eficiência das ventosas automáticas de abertura e fechamento não instantâneos como mecanismos para aliviar o golpe de aríete. As simulações foram realizadas utilizando o software UFC6 desenvolvido no Laboratório de Hidráulica Computacional (LHC) da UFC.

Palavras-chave: Ventosa, Golpe de aríete, UFC6.

\begin{abstract}
During transient phenomena in pipelines, very low pressures may occur, mostly in points of high elevations. In these cases, air accumulated in these points is carried out by water flow and way damage the pipeline. The most modern Waterhammer protection devices and among them, we find the air entry valves, which is the object of this work. In the present work, several Waterhammer pipeline simulations were performed in order to verify the performance of double effect automatic air valves, with non-instantaneous closing. The UFC6 computer software, developed in the Computational Hydraulic Laboratory (LHC) from UFC, were used to accomplish these simulations.
\end{abstract}

Key-words: Air valves, Waterhammer, UFC6.

\footnotetext{
Marcus Vinícius Sousa Rodrigues*

Engenheiro Mecânico. Doutorando em Engenharia Civil na área de concentração em Recursos Hídricos na Universidade Federal do Ceará - UFC. Professor Assistente do Departamento de Ciências Exatas, Tecnológicas e Humanas - DCETH da Universidade Federal Rural do Semi-Árido - UFERSA (Campus de Angicos - Angicos - RN).

Marco Aurélio Holanda de Castro

Engenheiro Civil, PhD, Drexel University - USA. Professor Titular do Departamento de Engenharia Hidráulica de Ambiental DEHA da Universidade Federal do Ceará - UFC (Campus dos Pici - Fortaleza - CE).

Marisete Dantas de Aquino

Engenheira de Pesca. Doutora, École des Haustes - França. Professora Associada do Departamento de Engenharia Hidráulica de Ambiental - DEHA da Universidade Federal do Ceará - UFC (Campus dos Pici - Fortaleza - CE).

*Endereço para correspondência: Universidade Federal Rural do Semi-Árido - UFERSA - Campus de Angicos - Departamento de Ciências Exatas, Tecnológicas e Humanas - DCETH. Angicos, RN, Brasil. CEP: 59515-000.

Tel: $+55(84) 3531-2472$

E-mail:marcus@ufersa.edu.br
} 


\section{Introdução}

Os sistemas hidráulicos, como um sistema para recalque de água, podem sofrer alterações nas condições de escoamento caracterizadas pela variação da pressão e da velocidade do fluido com o tempo, gerando regimes variados. Esses escoamentos em regimes variados são chamados de escoamentos transientes ou transitórios hidráulicos, e são caracterizados por ocorrer entre dois regimes permanentes.

As variações de pressão ao longo da tubulação podem ocorrer de forma muito brusca, ocasionando ruídos semelhantes a golpes de martelo, daí este fenômeno também ser conhecido comumente como golpe de aríete.

Durante os fenômenos transitórios podem ocorrer pressões muito baixas nos trechos mais elevados de um sistema hidráulico, ocorrendo o acúmulo de ar que é arrastado pela água, ocasionando sérios danos estruturais.

A presença de uma quantidade de ar na tubulação de um sistema de adução de água por recalque é a causa direta para a redução da seção e, consequentemente, tem-se a redução da capacidade de transporte, o que compromete seriamente o rendimento deste sistema. Este excesso de ar também pode ocasionar a elevação do consumo por sobrecarga da bomba ou uma redução da vazão.

No caso da adução por gravidade, o ar depositado nos pontos altos de um sistema hidráulico, geralmente os pontos onde ocorre a queda de pressão, acarreta um aumento da perda de carga e, consequentemente, uma redução da vazão de água.

Para um bom funcionamento e estabilidade de um sistema de adução, deve ser prevista a entrada de ar, pois, quando se esvazia uma tubulação, o ar deve entrar com o mesmo gradiente de pressão com o qual a água sai, de forma que a diferença de pressão entre o meio e o interior da tubulação permaneça dentro dos limites preestabelecidos, evitando o colapso por contração devido às subpressões.

Logo, para um perfeito funcionamento de um sistema de adução de água, deve-se instalar dispositivos que expulsem o ar acumulado e que admitam ar para evitar as contrações causadas pelas subpressões.

Os dispositivos mais modernos para o alívio do golpe de aríete trabalham com ar, os mais utilizados na prática são as ventosas automáticas, parte integrante deste trabalho. Devido ao fato de estes dispositivos trabalharem com ar, têm uma modelagem matemática bastante complexa.

\section{Metodologia}

Segundo Lessa (1984), podem ser feitas algumas hipóteses na simplificação do estudo das ventosas automáticas de duplo efeito. Pode-se supor que a vazão de ar através da ventosa é isoentrópica e as expansões e contrações do ar dentro da tubulação ocorrem isotermicamente. Além disso, assume-se que o volume de ar se mantém nas vizinhanças da válvula, sendo este volume pequeno quando comparado com o volume do líquido entre as seções.

A equação que governa a lei isotérmica dos gases é dada pela equação

$$
\mathrm{pV}=\mathrm{mRT}
$$

onde:

p (Pa) é a pressão absoluta no interior da tubulação;

$V\left(m^{3}\right)$ é o volume de ar;

$m(\mathrm{~kg})$ é a massa de ar;

$T(K)$ é a temperatura absoluta;

$R(\mathrm{~J} / \mathrm{kgK}$ ) é a constante do gás (para o ar tem-se que $R=287 \mathrm{~J} / \mathrm{kgK}$ ).

Na figura 1, é mostrada uma notação para ventosa. Desprezando as perdas na junção, tem-se 


$$
H_{p}(\mathrm{I}, \mathrm{K})=\mathrm{H}_{\mathrm{p}}(\mathrm{J}, 1)
$$

onde:

$H_{p}(I, K)$ é a carga hidráulica, em $m$;

na seção $K$ do trecho I no final do intervalo de tempo e $H_{p}(J, 1)$ é a carga hidráulica,

Em $m$, na seção 1 do trecho $J$ no final do intervalo de tempo.

Estando a ventosa fechada, tem-se:

$$
\mathrm{Q}_{\mathrm{p}}(\mathrm{I}, \mathrm{K})=\mathrm{Q}_{\mathrm{p}}(\mathrm{J}, 1)
$$

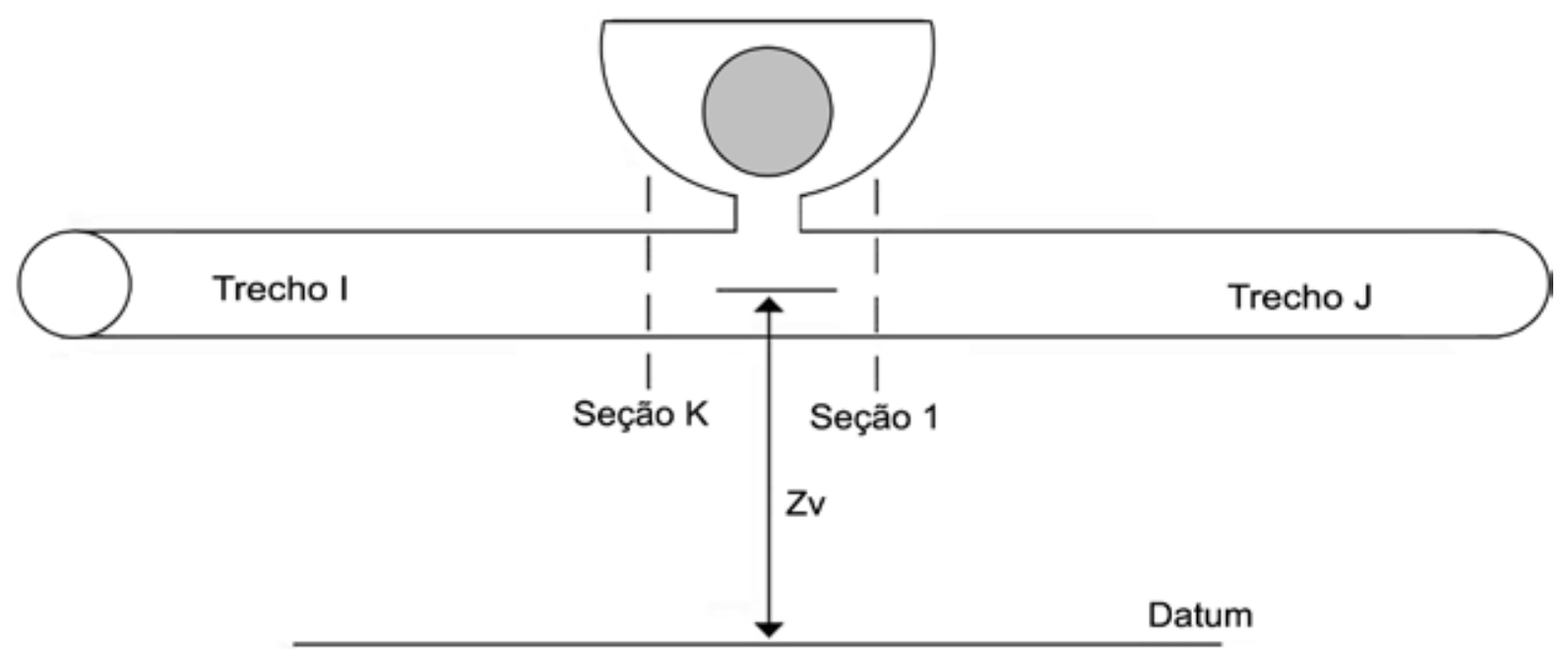

Figura 1 - Notação para ventosa

Quando a carga piezométrica cai abaixo da cota $Z_{V}(m)$ a válvula abre-se, permitindo a entrada de ar. Quando a carga volta a subir, ultrapassando $Z_{\mathrm{v}}$, então, o ar é expulso da tubulação.

Durante o funcionamento da ventosa, a equação da continuidade em termos do volume pode ser escrita da seguinte forma

$$
V_{\text {Par }}=V_{\mathrm{ar}}+0,5 \Delta t\left[Q_{p}(J, 1)+Q(J, 1)-Q_{p}(I, K)-Q(I, K)\right]
$$

onde:

$V_{P a r}$ é volume de ar na tubulação no final de intervalo de tempo;

$V_{a r}$ é $o$ volume de ar na tubulação no início do intervalo de tempo;

$Q(J, 1)$ e $Q_{P}(J, 1)$, são as vazões de água, em $\mathrm{m}^{3} / \mathrm{s}$, na seção 1 do trecho $J$ no início e no final do intervalo de tempo, respectivamente;

$Q(I, K)$ e $Q_{p}(I, K)$, são as vazões de água, em $\mathrm{m}^{3} / \mathrm{s}$, na seção $K$ do trecho I no início e no final do intervalo de tempo, respectivamente.

As equações características são válidas e podem ser expressas por comodidade computacional, como 


$$
\mathrm{C}^{+}: \mathrm{QP}_{\mathrm{P}}(\mathrm{I}, \mathrm{K})=\mathrm{C}_{\mathrm{P}}-\mathrm{C}_{\mathrm{a}} \mathrm{H}_{\mathrm{P}}(\mathrm{I}, \mathrm{K})
$$

$$
C^{-}: Q_{P}(J, 1)=C_{N}+C_{\mathrm{a}} H_{P}(J, 1)
$$

em que

$$
C_{p}=Q(I, K)+C_{a} H(I, K)-R \Delta t Q(I, K)|Q(I, K)|
$$

$$
\mathrm{C}_{\mathrm{N}}=\mathrm{Q}(\mathrm{J}, 1)-\mathrm{C}_{\mathrm{a}} \mathrm{H}(\mathrm{J}, 1)-\mathrm{R} \Delta \mathrm{t} \mathrm{Q}(\mathrm{J}, 1)|\mathrm{Q}(\mathrm{J}, 1)|
$$

onde:

$C_{a}\left(m^{2} s^{-1}\right)=g A / a$ e $R\left(m^{-3}\right)=f / 2 D A$ são constantes.

Aqui, a é a celeridade;

$g$ é a aceleração da gravidade;

fé o fator de atrito;

$A\left(m^{2}\right)$ e $D(m)$ são, respectivamente, a área e o diâmetro da seção do duto.

Da equação (2) tem-se que $H_{p}(I, K)=H_{p}(J, 1)$. Logo, substituindo as equações (5) e (6) na equação (4) e, em seguida, simplificando o resultado, pode-se escrever

$$
V_{\text {Par }}=\bar{D}+\bar{E} H_{p}(I, K)
$$

onde

$$
\overline{\mathrm{D}}=\mathrm{V}_{\mathrm{ar}}+0,5 \Delta \mathrm{t}\left[\mathrm{C}_{\mathrm{N}}-\mathrm{C}_{\mathrm{P}}+\mathrm{Q}(\mathrm{J}, 1)-\mathrm{Q}(\mathrm{I}, \mathrm{K})\right]
$$

$$
\overline{\mathrm{E}}=\mathrm{C}_{\mathrm{a}} \Delta \mathrm{t}
$$

Para pequenos intervalos de tempo, tem-se 


$$
m=m_{0}+0,5\left(\dot{m}_{0}+\dot{m}\right) \Delta t
$$

onde:

$m_{0}$ é massa de ar no início do intervalo de tempo;

$\dot{m}_{o}$ é a vazão em massa no início do intervalo de tempo;

e $\dot{m}$ é a vazão em massa no final do intervalo de tempo.

Substituindo as equações (9) e (12) na equação (1), tem-se o seguinte resultado

$$
p\left[\bar{D}+\overline{\mathrm{E}} \mathrm{H}_{\mathrm{P}}(\mathrm{I}, \mathrm{K})\right]=\left[\mathrm{m}_{0}+0,5 \Delta \mathrm{t}\left(\dot{\mathrm{m}}_{0}+\mathrm{m}\right)\right] \mathrm{R} \mathrm{T}
$$

onde:

p é a pressão do ar na ventosa.

A pressão absoluta p relaciona-se com a carga $\mathrm{H}_{\mathrm{p}}(\mathrm{I}, \mathrm{K})$ através da equação

$$
H_{\mathrm{P}}(\mathrm{I}, \mathrm{K})=\frac{\mathrm{P}}{\gamma}+\overline{\mathrm{F}}
$$

Sendo $\overline{\mathrm{F}}=\mathrm{Z}_{\mathrm{v}}-\mathrm{H}_{\mathrm{BV}}$, onde $\mathrm{H}_{\mathrm{BV}}(\mathrm{m})$ é a carga barométrica no local da ventosa e $\gamma\left(\mathrm{Nm}^{-3}\right)$ é o peso específico do líquido dentro do tubo. Substituindo a equação (14) na equação (13), pode-se chegar ao seguinte resultado

$$
\mathrm{p}\left(\overline{\mathrm{E}} \frac{\mathrm{p}}{\gamma}+\overline{\mathrm{D}}+\overline{\mathrm{EF}}\right)=\overline{\mathrm{H}}+\overline{\mathrm{G}} \mathrm{m}
$$

onde

$$
\overline{\mathrm{G}}=0,5 \Delta \mathrm{t} R \mathrm{~T} \text { e } \overline{\mathrm{H}}=\mathrm{m}_{0} \mathrm{R} \mathrm{T}+\overline{\mathrm{G}} \mathrm{m}_{0}
$$

Em termos de simplificação, pode-se escrever a equação (15) em termos do quociente p' = p/p $p_{0}, p_{0}$ é a pressão atmosférica local. Logo, a equação (15) assume a seguinte forma

$$
p^{\prime}\left(p^{\prime}+\bar{X}\right)=\bar{Y}+\bar{Z} \dot{m}
$$


$\overline{\mathrm{X}}=\frac{(\overline{\mathrm{D}}+\overline{\mathrm{EF}}) \gamma}{\overline{\mathrm{E}} \mathrm{p}_{0}}$

$\bar{Y}=\frac{\overline{\mathrm{H}} \gamma}{\mathrm{E} p_{0}^{2}}$

$\overline{\mathrm{Z}}=\frac{\overline{\mathrm{G}} \gamma}{\overline{\mathrm{E}} \mathrm{p}_{0}^{2}}$

Para resolver a equação (17) substitui-se a vazão em massa m que depende do regime de escoamento do ar. A vazão em massa através da ventosa é função da pressão atmosférica $p_{0}$, das temperaturas absolutas, externa $\mathrm{T}_{0}$ e interna $\mathrm{T}$, da pressão absoluta $\mathrm{p}$ e das relações $\mathrm{p} / \mathrm{p}_{0}$ e $\mathrm{p}_{0} / \mathrm{p}$. De acordo com Daily e Wylie há quatro zonas:

Zona 1: admissão de ar em regime crítico $\left(\mathrm{p}<0,528 \mathrm{p}_{0}\right)$ :

$$
\dot{m}=\frac{0,686 \mathrm{C}_{\text {in }} \mathrm{Apv}_{\mathrm{pv}} \mathrm{p}_{\mathrm{p}}}{\sqrt{\mathrm{RT}_{0}}}
$$

Zona 2: admissão de ar em regime subsônico $\left(0,528 \mathrm{p}_{0}<\mathrm{p}<\mathrm{p}_{0}\right)$ :

$$
\dot{\mathrm{m}}=\mathrm{C}_{\text {in }} \mathrm{A}_{\mathrm{PV}} \sqrt{7 \mathrm{p}_{0} \rho_{0}\left[\left(\frac{\mathrm{p}}{\mathrm{p}_{0}}\right)^{1,4286}-\left(\frac{\mathrm{p}}{\mathrm{p}_{0}}\right)^{1,714}\right]}
$$

Zona 3: descarga de ar em regime subsônico $\left(\mathrm{p}_{0}<\mathrm{p}<1,894 \mathrm{p}_{0}\right)$ :

$$
\dot{m}=-C_{\text {out }} A_{p V} p \sqrt{\frac{7}{R T}\left[\left(\frac{p_{0}}{p}\right)^{1,4286}-\left(\frac{p_{0}}{p}\right)^{1,714}\right]}
$$

Zona 4: descarga de ar em regime crítico ( $\left.\mathrm{p}>1,894 \mathrm{p}_{0}\right)$ :

$$
\dot{m}=-\frac{0,686 C_{\text {out }} A_{p v p}}{\sqrt{R T}}
$$


onde $\mathrm{C}_{\text {in }}$ e $\mathrm{C}_{\text {out }}$ são os coeficientes de descarga da ventosa para entrada e saída de ar, respectivamente; $\mathrm{A}_{\mathrm{PV}}$ é a área do orifício no final do intervalo de tempo; $\rho_{0}$ é a massa específica do ar; $\mathrm{T}_{0}$ é a temperatura absoluta externa da tubulação e $\mathrm{p}_{0}$ é a pressão atmosférica local (absoluta).

De acordo com as equações (21), (22), (23) e (24) pode-se verificar que a vazão em massa, $\dot{\mathrm{m}}$, é função do quociente $\mathrm{p}^{\prime}=\mathrm{p} / \mathrm{p}_{0}$, isto é, $\dot{\mathrm{m}}=\mathrm{f}\left(\mathrm{p}^{\prime}\right)$.

Segundo Lessa (1984), em muitas situações o intervalo de tempo adotado para efetuar os cálculos do transiente não é compatível com a suposição dada pela equação (12). Neste caso, torna-se necessária uma descrição de como se comportam a abertura e o fechamento do orifício da ventosa.

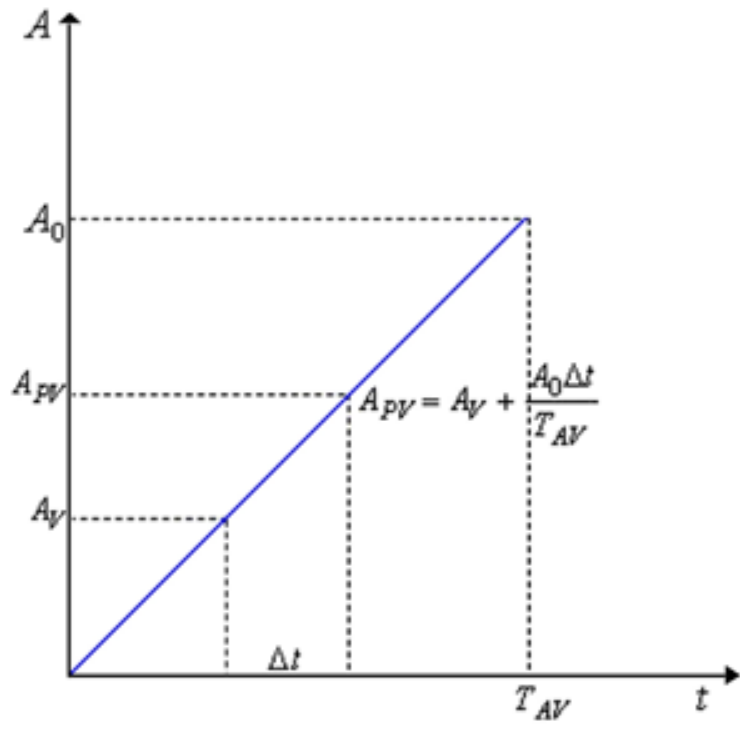

(a) Abertura da válvula ventosa

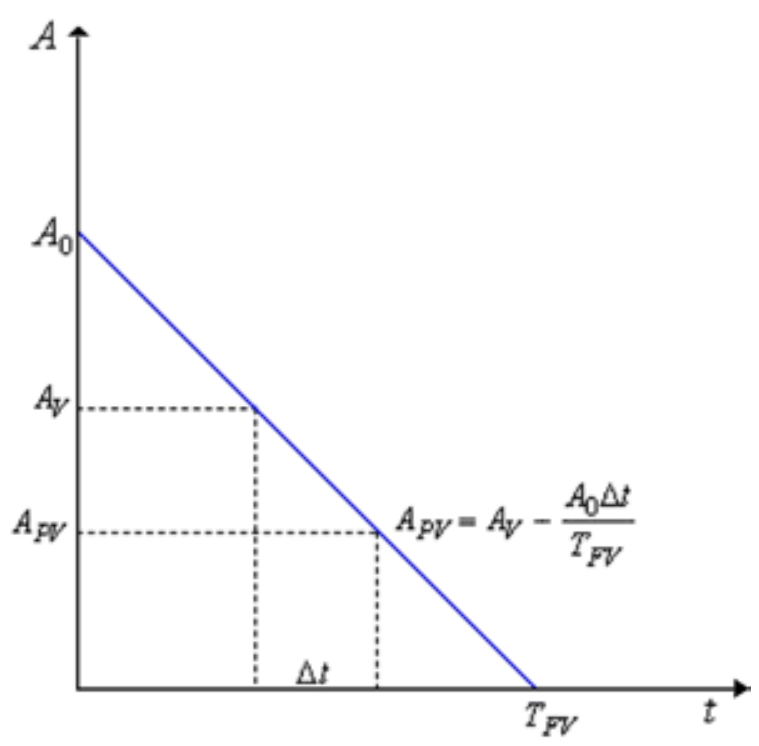

(b) Fechamento da válvula ventosa

Figura 2 - Abertura e fechamento da válvula ventosa

No presente trabalho foi feita a suposição, proposta por Lessa (1984), que a área do orifício da ventosa, $A_{v}$, deve variar linearmente de um intervalo de tempo para outro, seguindo as seguintes leis:

a) Para a abertura da ventosa

$$
A_{P V}=A_{V}+\left(\frac{\Delta t}{T_{A V}}\right) A_{C}
$$

No caso em que $A_{p v}>A_{0}$, faz-se $A_{p v}=A_{0}$.

b) Para o fechamento da ventosa

$$
A_{P V}=A_{V}-\left(\frac{\Delta t}{T_{F V}}\right) A_{C}
$$

No caso em que $A_{p v}<A_{0}$, faz-se $A_{p v}=0$.

Tem-se que $A_{v}$ é a área do orifício no início do intervalo de tempo; $A_{0}$ é a área do orifício totalmente 
aberto; $\mathrm{T}_{\mathrm{AV}}$ é o tempo de abertura do orifício e $\mathrm{T}_{\mathrm{FV}}$ é o tempo de fechamento do orifício.

Na figura 2 pode ser visto, graficamente, como a área do orifício da ventosa varia linearmente com o tempo, tanto na abertura (figura 2.a) quanto no fechamento (figura 2.b) da válvula ventosa.

\section{Simulações e resultados}

Para este trabalho, foi utilizado um trecho da Adutora Sertaneja, proposto por Barbosa (2006), como perfil para as simulações realizadas. Para realizar as simulações, foi utilizado o programa de transiente hidráulico, UFC6, que faz parte do Sistema UFC para simulação hidráulica de redes de abastecimento de água, de esgoto e adutoras, desenvolvido no Laboratório de Hidráulica Computacional (LHC) da Universidade Federal do Ceará, sob a orientação do professor PhD Marco Aurélio Holanda de Castro.

No programa UFC6 constam os seguintes mecanismos para o alívio do golpe de aríete: Tanque de Alimentação Unidirecional (TAU), Chaminé de Equilíbrio, e as Ventosas Automáticas de Duplo Efeito (VADE). As VADEs dividem-se em duas categorias: as ventosa automáticas de duplo efeito com abertura e fechamento instantâneos (teóricas), e as ventosas automáticas com abertura e fechamento não instantâneos (reais), cuja implementação faz parte deste trabalho.

As simulações aplicadas ao perfil proposto têm como principal objetivo analisar o comportamento das ventosas de abertura e fechamento não instantâneos, além de fazer um comparativo de seu funcionamento com as ventosas de abertura e fechamento instantâneos.

\section{Problema hidráulico}

O programa UFC6 apresenta as condições hidráulicas de trabalho através de envoltórias de pressões máximas e mínimas (figura 3). O problema proposto apresenta uma bomba hidráulica na extremidade de montante, um reservatório com nível constante na extremidade de jusante e, entre eles, os demais nós considerados como junções.

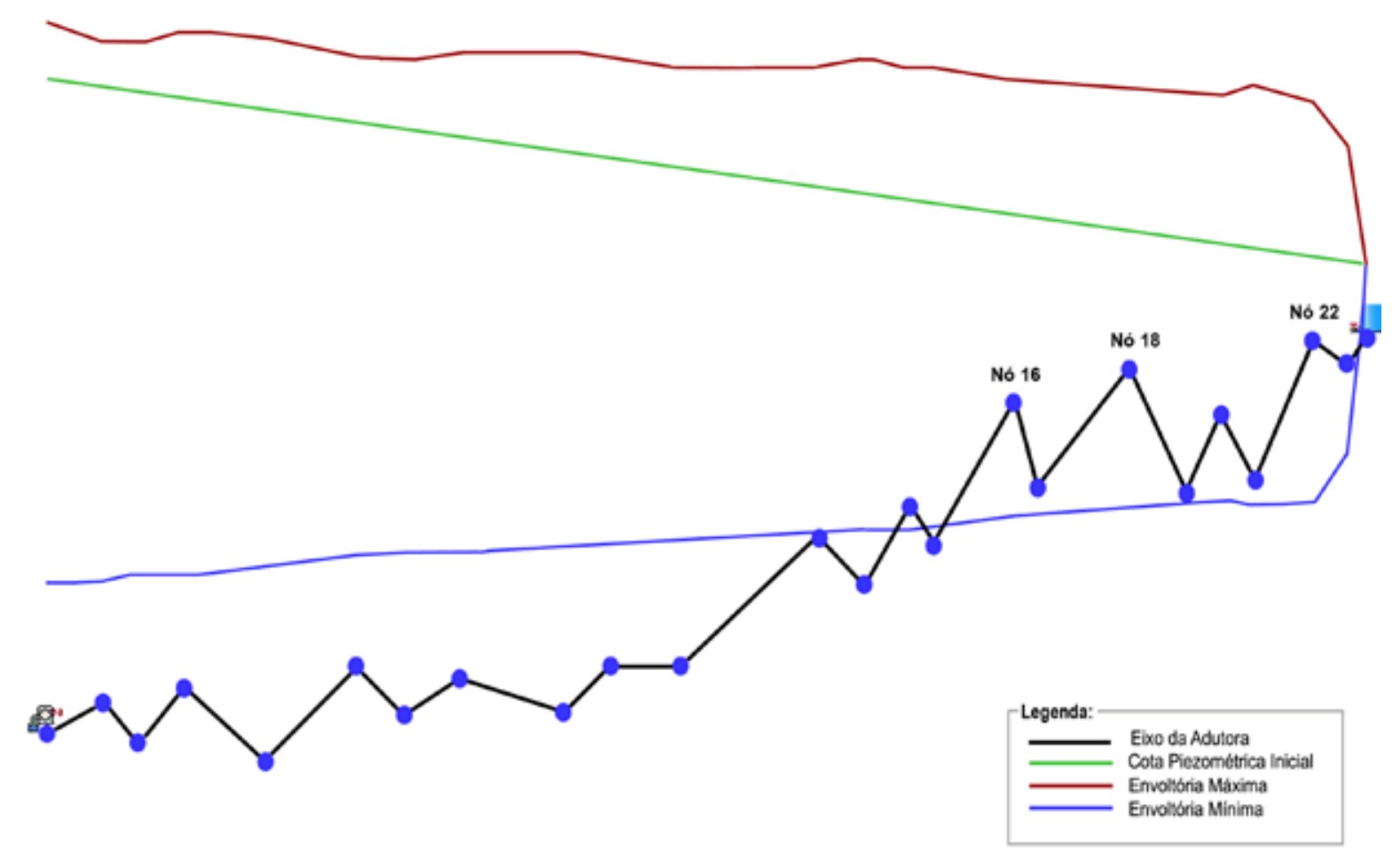

Figura 3 - Perfil do problema hidráulico (Fonte: Software UFC6) 


\begin{tabular}{|c|c|c|c|c|c|c|}
\hline Nó & $\mathrm{H}(\mathrm{m})$ & $\operatorname{Hmax}(\mathrm{m})$ & $\operatorname{Hmin}(\mathrm{m})$ & $P$ (mca) & Pmax (mca) & Pmin (mca) \\
\hline 1 & 439,00 & 455,23 & 296,56 & 195,00 & 201,23 & 42,56 \\
\hline 2 & 436,71 & 449,44 & 297,41 & 174,01 & 186,74 & 34,71 \\
\hline 3 & 435,33 & 449,05 & 298,90 & 184,13 & 197,85 & 47,70 \\
\hline 4 & 433,49 & 452,43 & 298,90 & 166,49 & 185,43 & 31,90 \\
\hline 5 & 430,28 & 450,56 & 300,84 & 184,28 & 204,56 & 54,84 \\
\hline 6 & 426,61 & 445,15 & 304,53 & 153,61 & 172,15 & 31,53 \\
\hline 7 & 424,78 & 444,42 & 305,53 & 165,58 & 185,22 & 46,33 \\
\hline 8 & 422,48 & 445,95 & 305,35 & 152,98 & 176,45 & 36,85 \\
\hline 9 & 418,36 & 446,67 & 307,12 & 158,36 & 186,67 & 47,13 \\
\hline 10 & 416,52 & 445,12 & 307,13 & 143,52 & 172,12 & 34,72 \\
\hline 11 & 413,77 & 441,64 & 309,18 & 140,77 & 168,64 & 36,18 \\
\hline 12 & 408,26 & 442,71 & 311,00 & 98,56 & 133,01 & 1,30 \\
\hline 13 & 406,43 & 444,71 & 311,67 & 110,33 & 148,63 & 15,57 \\
\hline 14 & 404,59 & 442,01 & 312,02 & 86,09 & 123,51 & $-6,48$ \\
\hline 15 & 403,58 & 442,33 & 312,55 & 96,18 & 134,83 & 5,05 \\
\hline 16 & 400,46 & 438,77 & 315,56 & 52,96 & 91,27 & $-31,94$ \\
\hline 17 & 399,55 & 438,63 & 315,89 & 76,05 & 115,13 & $-7,61$ \\
\hline 18 & 395,88 & 436,42 & 317,89 & 38,68 & 79,22 & $-39,31$ \\
\hline 19 & 393,58 & 435,63 & 319,06 & 71,78 & 113,83 & $-2,74$ \\
\hline 20 & 392,21 & 434,01 & 320,14 & 48,01 & 89,81 & $-24,06$ \\
\hline 21 & 390,83 & 437,25 & 318,64 & 65,13 & 111,55 & $-7,06$ \\
\hline 22 & 388,54 & 432,33 & 319,39 & 23,54 & 87,33 & -45.61 \\
\hline 23 & 387,18 & 420,05 & 333,30 & 28,66 & 61,55 & $-25,30$ \\
\hline 24 & 386,43 & 386,43 & 386,43 & 20,43 & 20,43 & 20,43 \\
\hline
\end{tabular}

Tabela 1 - Cargas e pressões referentes ao problema hidráulico (Fonte: Software UFC6)

Na tabela 1 são apresentados os valores da carga hidráulica e pressão, sem o mecanismo de alívio, para cada nó. Pode-se ver claramente que nos nós 16, 18 e 22, são os nós que apresentam as pressões negativas mais baixas, respectivamente, - 31,94 mca, - 39,31 mca e - 45,61 mca, valores estes que podem causar sérios danos e levar a tubulação a um colapso por contração.

A tabela 1 e a figura 3 também mostram que os maiores valores para pressões máximas ocorrem nos nós próximos à bomba. 0 maior valor de pressão máxima ocorre no nó 5, cujo valor é de 204,56 mca.

Logo, de acordo com a figura 3 e os dados apresentados na tabela 1, pode-se chegar à conclusão que o grande problema desta adutora está relacionado com as pressões negativas. Então, serão propostas simulações, objetivando uma solução hidráulica eficiente para o problema.

Uma solução hidráulica eficiente deve elevar as pressões mínimas a valores aceitáveis, além de reduzir as pressões máximas.

\section{Soluções hidráulicas}

Serão apresentadas duas soluções hidráulicas para este problema. A primeira solução refere-se à aplicação de ventosas automáticas de duplo efeito com abertura e fechamento instantâneos (ventosas teóricas) e a segunda solução refere-se à aplicação das ventosas automáticas de duplo efeito com abertura e fechamento não instantâneos (ventosas reais).

As ventosas devem ser aplicadas nos pontos altos da adutora, visto que nestes locais ocorre o acúmulo de ar, provocando cavidades e diminuindo a eficiência do escoamento. O diâmetro de cada ventosa está 
relacionado diretamente com o diâmetro da tubulação, como mostra a tabela 2.

\begin{tabular}{|c|c|}
\hline Diâmetro da tubulação $(\mathrm{mm})$ & Diâmetro da ventosa $(\mathrm{mm})$ \\
\hline $80-250$ & 50 \\
\hline $300-400$ & 80 \\
\hline $450-550$ & 100 \\
\hline $600-1200$ & 150 \\
\hline $1400-2400$ & 200
\end{tabular}

Tabela 2 - Relação entre diâmetros da ventosa e da tubulação (Fonte: ARI Flow Control Accesories)

Na primeira solução hidráulica aplicam-se ventosas automáticas de duplo efeito com abertura e fechamento instantâneos (ventosas teóricas) nos nós 16, 18 e 22. As ventosas utilizadas são idênticas e possuem as seguintes características: diâmetros de $50 \mathrm{~mm}$, a carga barométrica local é de 10,3 m, as temperaturas externas e internas são, respectivamente, $26{ }^{\circ} \mathrm{C}(299,15 \mathrm{~K})$ e $27{ }^{\circ} \mathrm{C}(300,15 \mathrm{~K})$ e a constante dos gases para o ar é $\overline{\mathrm{R}}=287 \mathrm{~J} / \mathrm{kg} . \mathrm{K}$.

No momento em que ocorre a primeira onda de sobrepressão, as ventosas não são afetadas, portanto, não há cavidade e as mesmas encontram-se fechadas. Porém, quando a primeira onda de subpressão atinge o ponto onde se localizam as ventosas, estas se abrem permitindo a entrada de ar na tubulação suavizando a queda da pressão.

Após certo período de tempo, uma nova onda de sobrepressão atinge as ventosas, então, o ar que estava aprisionado é expulso. No momento que a ventosa fecha e ocorre a rejunção das colunas de água, a pressão se eleva a valores maiores que os observados na primeira coluna de líquido. Logo, a onda de subpressão seguinte também será mais intensa que a primeira.

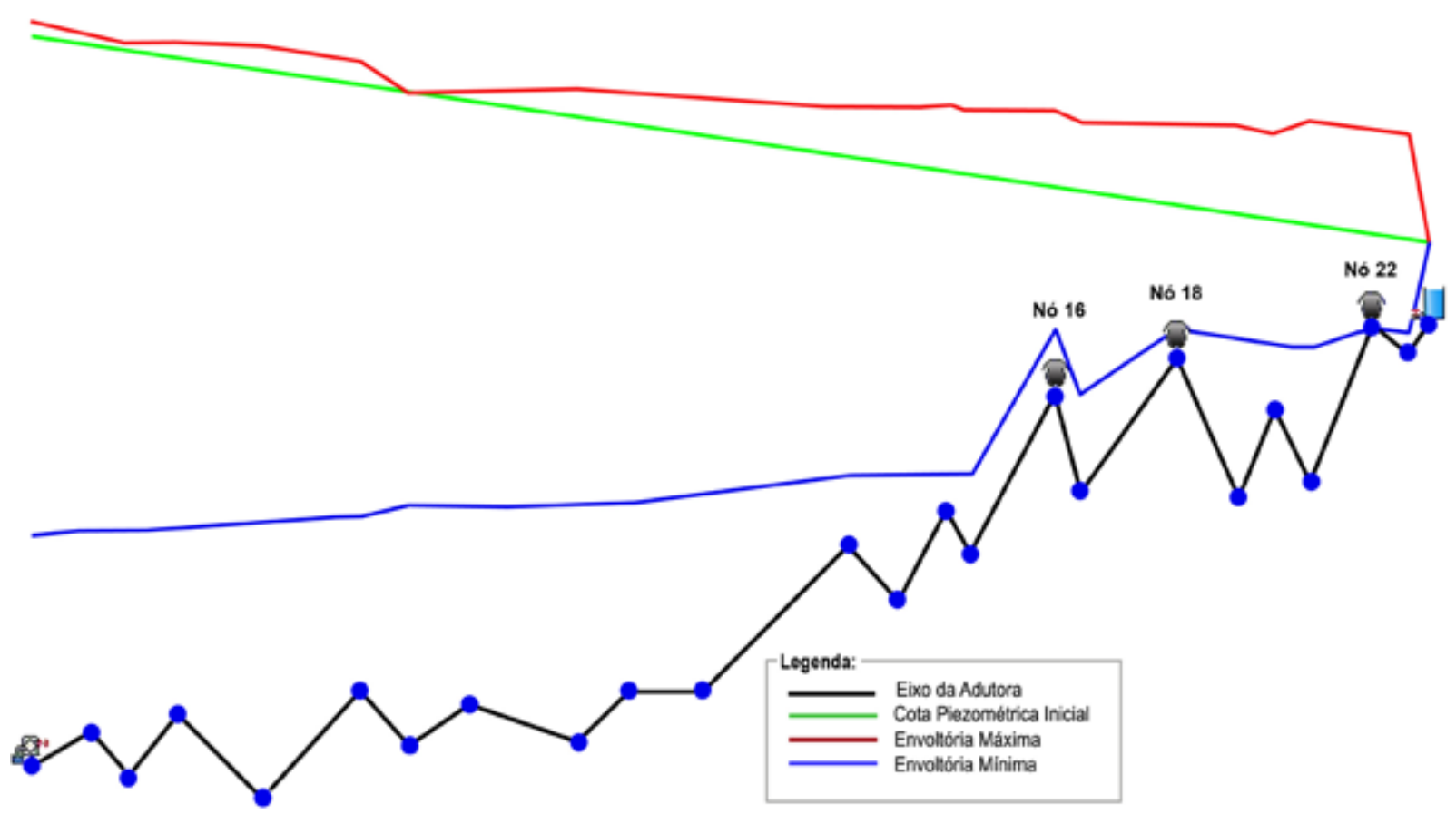

Figura 4 - Perfil referente à primeira solução hidráulica (Fonte: Software UFC6) 
A figura 4 mostra o perfil da primeira solução hidráulica. Nesta figura pode-se observar que a eficiência deste conjunto é bastante satisfatória, pois, além de elevar a envoltória de mínima, ocorre o alívio das pressões de máxima, tendo a envoltória de máxima se aproximando da linha piezométrica inicial.

Logo em seguida são apresentados, na tabela 3, os valores obtidos para a carga hidráulica e pressão para cada nó.

\begin{tabular}{|c|c|c|c|c|c|c|}
\hline Nó & $\mathrm{H}(\mathrm{m})$ & $\operatorname{Hmax}(\mathrm{m})$ & $\operatorname{Hmin}(\mathrm{m})$ & $P$ (mca) & Pmax (mca) & Pmin (mca) \\
\hline 1 & 439,00 & 442,37 & 312,08 & 195,00 & 188,37 & 58,08 \\
\hline 2 & 436,71 & 439,38 & 313,82 & 174,01 & 176,68 & 51,12 \\
\hline 3 & 435,33 & 436,78 & 313,48 & 184,13 & 185,58 & 62,28 \\
\hline 4 & 433,49 & 436,65 & 314,37 & 166,49 & 169,65 & 47,37 \\
\hline 5 & 430,28 & 436,29 & 315,55 & 184,28 & 190,29 & 69,55 \\
\hline 6 & 426,61 & 432,08 & 317,12 & 153,61 & 159,08 & 44,12 \\
\hline 7 & 424,78 & 424,78 & 319,97 & 165,58 & 165,58 & 60,77 \\
\hline 8 & 422,48 & 425,59 & 319.35 & 152,98 & 156,09 & 49,85 \\
\hline 9 & 418,36 & 425,16 & 320,37 & 158,36 & 165,16 & 60,37 \\
\hline 10 & 416,52 & 424,47 & 320,35 & 143,52 & 151,47 & 47,35 \\
\hline 11 & 413,77 & 423,16 & 323,48 & 140,77 & 150,16 & 50,48 \\
\hline 12 & 408,26 & 420,35 & 327,30 & 98,56 & 110,65 & 17,60 \\
\hline 13 & 406,43 & 420,43 & 327,10 & 110,33 & 124,33 & 31,00 \\
\hline 14 & 404,59 & 421,06 & 328,08 & 86,09 & 102,56 & 9,58 \\
\hline 15 & 403,58 & 419,61 & 327,49 & 96,18 & 112,11 & 19,99 \\
\hline 16 & 400,46 & 419,64 & 364,52 & 52,96 & 72,14 & 17,02 \\
\hline 17 & 399,55 & 417,15 & 347,97 & 76,05 & 93,65 & 24,47 \\
\hline 18 & 395,88 & 416,69 & 364,46 & 38,68 & 59,49 & 7,26 \\
\hline 19 & 393,58 & 415,66 & 361,63 & 71,78 & 93,86 & 39,83 \\
\hline 20 & 392,21 & 413,87 & 360,63 & 48,01 & 69,67 & 16,43 \\
\hline 21 & 390,83 & 417,37 & 359,60 & 65,13 & 91,67 & 33,90 \\
\hline 22 & 388,54 & 415,26 & 364,79 & 23,54 & 50,26 & $-0,21$ \\
\hline 23 & 387,18 & 413,60 & 362,98 & 28,66 & 55,10 & 4,48 \\
\hline 24 & 386,43 & 386,43 & 386,43 & 20,43 & 20,43 & 20,43 \\
\hline
\end{tabular}

Tabela 3 - Cargas e pressões referentes a primeira solução hidráulica (Fonte: Software UFC6)

Através da tabela 3, pode-se observar que as pressões mínimas nos nós 16, 18 e 22 assumem os seguintes valores: 17,02 mca, 7,26 mca e - 0,21 mca, respectivamente. Também, verifica-se que no nó 5 a pressão foi aliviada, assumindo um valor de 190,29 mca.

Na figura 5, é mostrado como a carga varia no nó 22 durante os 60 segundos do cálculo do transiente. Pode-se ver que a pressão máxima assume o valor de 50,26 mca, enquanto a pressão mínima assume o valor de -0,21 mca.

De acordo com Peroba Junior (2007), este tipo de ventosa é bastante eficiente para resolver este problema hidráulico. Os dados apresentados na tabela 4 mostram a eficiência das ventosas.

Agora, aplica-se a segunda solução hidráulica. Nesta solução são aplicadas ventosas automáticas de duplo efeito com abertura e fechamento não instantâneos (ventosas reais) nos nós 16, 18 e 22. As ventosas utilizadas são idênticas e possuem as seguintes características: diâmetro de $50 \mathrm{~mm}$, a carga barométrica local é de $10,3 \mathrm{~m}$, as temperatura externa e interna são, respectivamente, $26{ }^{\circ} \mathrm{C}$ e $27^{\circ} \mathrm{C}$ e 
a constante dos gases para o ar é $\overline{\mathrm{R}}=287 \mathrm{~J} / \mathrm{kg} . \mathrm{K}$.

Neste tipo de ventosa, foi considerada uma metodologia em que tanto a abertura quanto o fechamento não ocorrem instantaneamente, ou seja, foi considerado um tempo para a abertura de 5 segundos e um tempo para o fechamento de 10 segundos.

Quando a primeira onda de subpressão atinge os pontos onde estão instaladas as ventosas, estas se abrem (a abertura ocorre no período de tempo de 5 segundos) permitindo a entrada de ar, aliviando a queda de pressão. Em seguida, quando uma onda de sobrepressão atinge as ventosas, estas passam a se fechar (o fechamento ocorre no período de tempo de 10 segundos) provocando a expulsão do ar aprisionado no interior da tubulação.

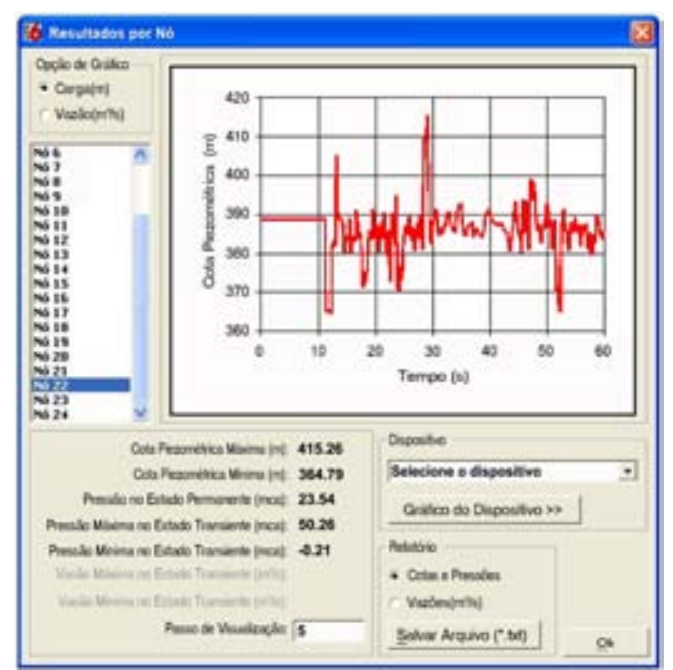

Figura 5 - Variação da carga no nó 22 referente à primeira solução hidráulica (Fonte: Software UFC6)

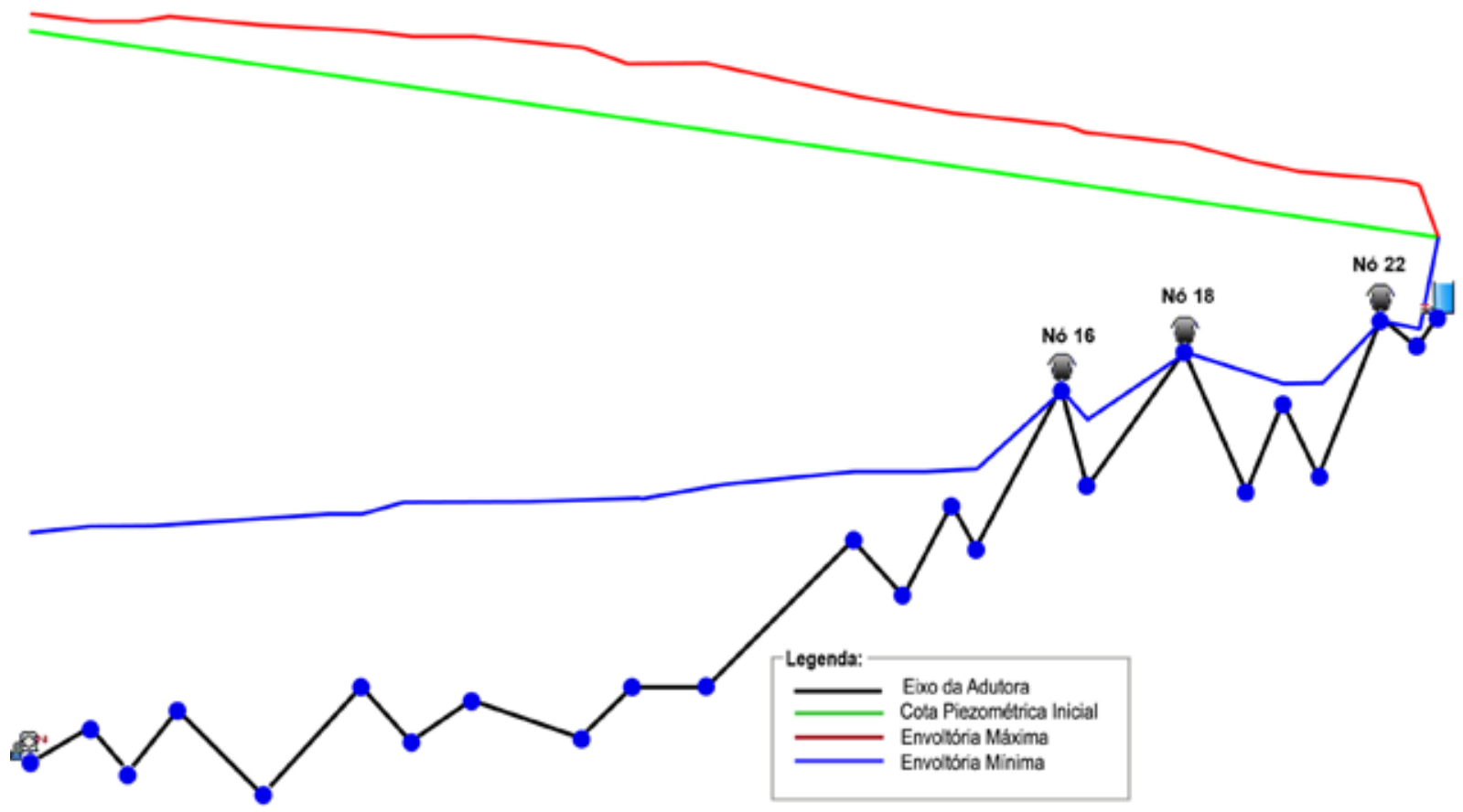

Figura 6 - Perfil referente à segunda solução hidráulica (Fonte: Software UFC6) 
Na figura 6, pode-se ver, semelhantemente à primeira solução, que a envoltória de mínima elevou-se, enquanto a envoltória de máxima aproxima-se da carga piezométrica inicial. Em seguida, tem-se na tabela 4 os valores para a carga e a pressão em cada nó, para a segunda solução hidráulica.

\begin{tabular}{|c|c|c|c|c|c|c|}
\hline Nó & $\mathrm{H}(\mathrm{m})$ & $\operatorname{Hmax}(\mathrm{m})$ & $\operatorname{Hmin}(\mathrm{m})$ & P (mca) & Pmax (mca) & Pmin (mca) \\
\hline 1 & 439,00 & 443,21 & 312,08 & 195,00 & 189,21 & 58,08 \\
\hline 2 & 436,71 & 441,04 & 313,84 & 174,01 & 178,34 & 51,14 \\
\hline 3 & 435,33 & 440,73 & 313,48 & 184,13 & 189,53 & 62,28 \\
\hline 4 & 433,49 & 442,45 & 314,37 & 166,49 & 175,45 & 47,37 \\
\hline 5 & 430,28 & 439,85 & 315,55 & 184,28 & 193,83 & 69,55 \\
\hline 6 & 426,61 & 438,40 & 317,12 & 153,61 & 165,40 & 44,12 \\
\hline 7 & 424,78 & 437,23 & 319,97 & 165,58 & 178,03 & 60,77 \\
\hline 8 & 422,48 & 436,77 & 319,35 & 152,98 & 167,27 & 49,85 \\
\hline 9 & 418,36 & 434,20 & 320,37 & 158,36 & 174,20 & 60,37 \\
\hline 10 & 416,52 & 429,80 & 320,35 & 143,52 & 156,80 & 47,35 \\
\hline 11 & 413,77 & 429,95 & 323,48 & 140,77 & 156,95 & 50,48 \\
\hline 12 & 408,26 & 421,78 & 327,30 & 98,56 & 112,08 & 17,60 \\
\hline 13 & 406,43 & 420,30 & 327,10 & 110,33 & 124,20 & 31,00 \\
\hline 14 & 404,59 & 417,76 & 328,08 & 86,09 & 99,26 & 9,58 \\
\hline 15 & 403,58 & 417,38 & 327,49 & 96,18 & 109,88 & 19,99 \\
\hline 16 & 400,46 & 414,60 & 347,03 & 52,96 & 67,10 & $-0,47$ \\
\hline 17 & 399,55 & 412,57 & 340,63 & 76,05 & 89,07 & 17,13 \\
\hline 18 & 395,88 & 410,11 & 356,93 & 38,68 & 52,91 & $-0,27$ \\
\hline 19 & 393,58 & 406,03 & 352,66 & 71,78 & 84,23 & 30,86 \\
\hline 20 & 392,21 & 403,64 & 349,65 & 48,01 & 59,44 & 5,45 \\
\hline 21 & 390,83 & 402,67 & 34,921 & 65,13 & 76,97 & 23,51 \\
\hline 22 & 388,54 & 401,12 & 364,75 & 23,54 & 36,12 & $-0,25$ \\
\hline 23 & 387,18 & 399,33 & 362,94 & 28,66 & 40,83 & 4,44 \\
\hline 24 & 386,43 & 386,43 & 386,43 & 20,43 & 20,43 & 20,43 \\
\hline
\end{tabular}

Tabela 4 - Cargas e pressões referentes à segunda solução hidráulica (Fonte: Software UFC6)

Analisando os dados da tabela 4, verifica-se que a pressão mínima nos nós 16, 18 e 22 assumem, respectivamente, os valores - 0,47 mca, - 0,27 mca e - 0,25 mca, valores estes que podem ser considerados satisfatórios. Enquanto a pressão máxima no nó 5 assume um valor de 193,83 mca.

Na figura 7, pode-se ver como varia a carga no nó 22 durante os 60 segundos do cálculo do transiente. Pode-se ver, nesta figura, que a pressão máxima é de 36,12 mca, enquanto a pressão mínima é de - 0,25 mca.

Na tabela 5, será apresentado um comparativo para os menores valores para pressões mínimas e os valores para pressões máximas para a primeira solução (ventosas teóricas) e segunda solução (ventosas reais).

Analisando os dados da tabela 5, pode-se concluir que, referente às pressões mínimas, a maior diferença ocorre no nó 16, para a primeira solução a pressão mínima passa a assumir o valor de 17,02 mca, enquanto na segunda solução este valor é de - 0,47 mca. Em relação às pressões máximas, tem-se que no nó 3 ocorre a maior diferença entre as duas soluções, para a primeira solução o valor é de 185,58 mca, enquanto na segunda solução este valor é de 189,53. 


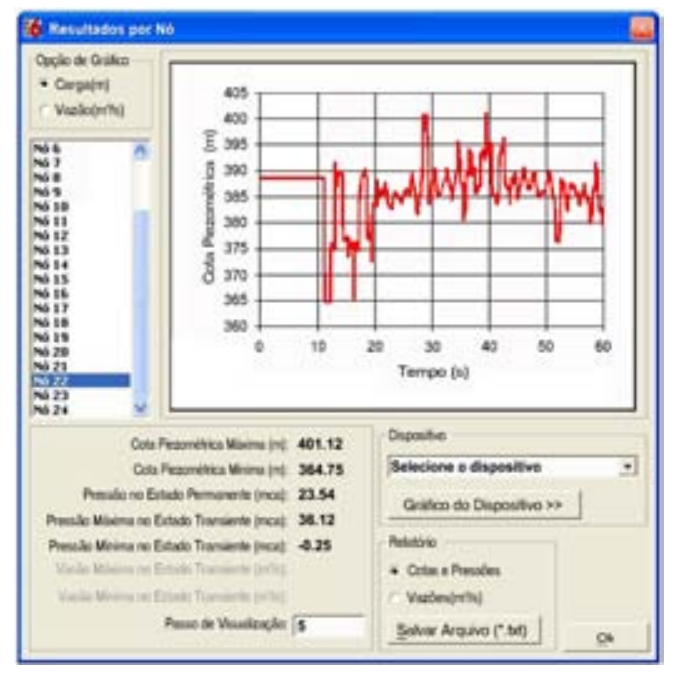

Figura 7 - Variação da carga no Nó 22 referente à segunda solução hidráulica (Fonte: Software UFC6)

\begin{tabular}{|c|c|c|c|}
\hline \multicolumn{4}{|c|}{ Pressão mínima } \\
\hline Nó & Problema hidráulico & Primeira solução & Segunda solução \\
\hline 16 & - 31,94 mca & $17,02 \mathrm{mca}$ & $-0,47 \mathrm{mca}$ \\
\hline 18 & - 39,31 mca & $7,26 \mathrm{mca}$ & $-0,27 \mathrm{mca}$ \\
\hline 22 & - 45,61 mca & $-0,21 \mathrm{mca}$ & $-0,25 \mathrm{mca}$ \\
\hline \multicolumn{4}{|c|}{ Pressão máxima } \\
\hline Nó & Problema hidráulico & Primeira solução & Segunda solução \\
\hline 1 & $201,23 \mathrm{mca}$ & $188,37 \mathrm{mca}$ & $189,21 \mathrm{mca}$ \\
\hline 3 & $197,85 \mathrm{mca}$ & $185,58 \mathrm{mca}$ & $189,53 \mathrm{mca}$ \\
\hline 5 & $204,56 \mathrm{mca}$ & $190,29 \mathrm{mca}$ & $193,83 \mathrm{mca}$ \\
\hline
\end{tabular}

Tabela 5 - Comparativo entre as soluções hidráulicas para pressões mínimas e máximas (Fonte: Software UFC6)

Na figura 8, pode-se ver as duas soluções hidráulicas sobrepostas sobre o problema hidráulico. De acordo com esta figura, pode-se ver que, próximo à bomba a primeira solução alivia melhor as pressões máximas, enquanto que próximo ao reservatório ocorre o inverso. Também, pode-se ver que as envoltórias de mínimas têm valores bem próximos até aproximadamente o nó 16, então, a partir daí tem-se que a eficiência para a primeira solução foi melhor.

Através da figura 8, pode-se ver que ambas as soluções são eficientes para resolver o problema hidráulico. Isto é, em ambos os casos, tanto a envoltória de mínima se elevou, como a envoltória de máxima foi aliviada.

\section{Conclusões}

Foram propostas duas soluções hidráulicas para o problema proposto: uma solução envolvendo a aplicação de ventosas automáticas de duplo efeito com abertura e fechamento instantâneos (ventosas teóricas) e outra envolvendo a aplicação de ventosas automáticas de duplo efeito com abertura e fechamento não instantâneos (ventosas reais).

O intuito do trabalho consiste em mostrar a eficiência de se aplicar ventosas automáticas de duplo 


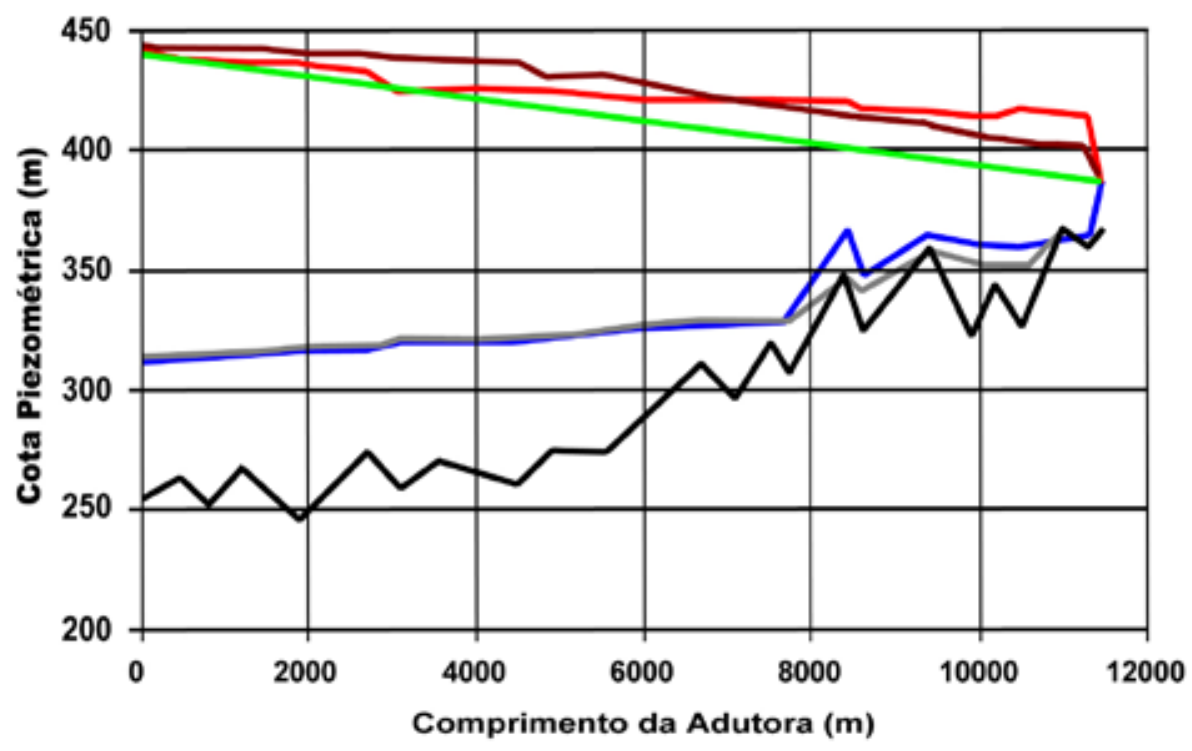

— Envoltória de pressões máximas (primeira soluçăo)

- Envoltória de pressōes mínimas (primeira solução)

— Envoltória de pressões máximas (segunda solução)

— Envoltória de pressōes mínimas (segunda solução)

- Linha piezométrica permanente

- Perfil do terreno

Figura 8 - Envoltórias de pressões das soluções hidráulicas (Fonte: Software UFC6)

efeito com abertura e fechamento não instantâneos, como mecanismo de alívio do golpe de aríete.

Em trabalhos anteriores, foi mostrado que a aplicação das ventosas automáticas de duplo efeito com abertura e fechamento instantâneos como mecanismo de alívio para o golpe de aríete obtém resultados satisfatórios, o que implica que esse tipo de ventosa é bem eficiente para este propósito. Neste trabalho também foi confirmado este fato, através da primeira solução hidráulica.

Uma segunda solução hidráulica foi proposta, através da utilização de ventosas automáticas de duplo efeito com abertura e fechamento não instantâneos. Nesta solução, também mostrou resultados satisfatórios, o que implica que esse tipo de ventosa também é muito eficiente como mecanismo de alívio do golpe de aríete.

Foi feito um comparativo, tanto através de figura como tabela de valores, fornecidos pelo Software UFC6, no cálculo do transiente, que as duas soluções forneceram resultados satisfatórios.

Verificou-se também que houve uma pequena diferença de valores nas duas soluções. A principal razão para a ocorrência desta diferença deve-se ao fato de que a ventosa proposta na metodologia deste trabalho tem a sua área do orifício variando linearmente com o tempo.

\section{Referências Bibliograficas}

BARBOSA, M. P. R. (2006) Modelagem Computacional de Chaminé de Equilíbrio e Tanques de Alimentação Unidirecional como Mecanismo de Alívio do Golpe de Aríete em Adutoras. Dissertação de Mestrado, DEHA/ UFC.

CASTRO, M. A. H. de. O sistema UFC para simulação hidráulica de redes de abastecimento de água, de esgoto e adutoras. In: GOMES, H. P.; GARCÍA, R. P.; REY, P. L. I. (orgs.). Abastecimento de água - O estado da arte e técnicas avançadas. João Pessoa: Editora Universitária - UFPB, 2007. 386 p.

CHAUDRY, M. H. (1979) Applied Hydraulic Transients. Van Nostrand Reinhold Company, New York, $28^{\mathrm{a}}$ edição.

KOELLE, E. (1983) Transientes Hidráulicos em Instalações de Condutos Forçados. Tese de Livre Docência, 
EPUSP, São Paulo.

LESSA, R. C. (1984) Transientes Hidráulicos em Sistemas Complexos de Adução de Água. Dissertação de Mestrado, ESSC/USP, 1984.

LESSA, R. C. (1990) Análise do Funcionamento de Acessórios durante a Ocorrência de Transitórios Hidráulicos. Tese de Doutorado, ESSC/USP.

LESSA, R. C.; RIGHETTO, A. M. (1983) Influência das Ventosas nos Transientes Hidráulicos. $12^{\circ}$ Congresso Brasileiro de Engenharia Sanitária e Ambiental, Camburiú, S. C. ABES, pp. 50-65.

NEIVA, R. M. S. (2000) Modelagem Computacional do Golpe de Aríete em Adutoras. Dissertação de mestrado, DEHA - UFC.

PEROBA JUNIOR, F. (2007) Modelagem Computacional de Ventosas Automáticas de Duplo Efeito como Mecanismo de Alívio do Golpe de Aríete. Dissertação de Mestrado, DEHA/UFC.

RIGHETTO, A. M. (1972) Considerações sobre o Golpe de Aríete em Instalações Hidráulicas. Dissertação de Mestrado. ESSC/USP.

STREETER, V. L.; WYLIE, E. B. (1978) Fluid Transients. MacGrawHill Editora.

STREETER, V. L.; WYLIE, E. B. (1982) Mecânica dos Fluidos. MacGrawHill do Brasil, 585p.

STREETER, V. L., WYLIE, E. B. (1967) Hydraulic Transients. MacGrawHill Book Co., New York.

TULLIS, J. P., STREETER, V. L. WYLIE, E. B. (1976) Waterhammer Analysis with air release. Proceeding of the second international conference on pressure surges, BHRA, London, UK, pp. 35-47.

VIANNA, M. R. Hidráulica Básica Aplicada aos Sistemas de Abastecimento de Água. (1995) Vol. 1, Belo Horizonte, Instituto de Engenharia Aplicada Editora, 300p. 